\title{
NLR KINEMATICS IN CSS RADIO SOURCES
}

\author{
RICHARD GELDERMAN \\ National Research Council, NASA/GSFC, Code 681 \\ Laboratory for Astronomy and Solar Physics, Greenbelt, MD
}

Compact Steep-Spectrum (CSS) radio sources are defined by their powerful yet subgalactic radio structure. High resolution radio interferometers reveal subarcsecond double, triple, and core-jet radio morphologies resembling miniature FR II sources. There are three promising scenarios for how such powerful radio sources could be so intrinsically small: 1) they are young sources which have not had time to grow large, 2) they are confined sources with exceptionally dense gas in the host which either smothers an existing source or frustrates its initial growth, or 3) they are enhanced sources with an intrinsic power much lower than one might expect based on their radio luminosity. To investigate these scenarios, we are continuing a comprehensive optical study of a sample of Compact Steep Spectrum objects.

From the radio selected CSS sources of Spencer et al. (1989) and Fanti et al. (1990) we have observed objects with $\mathrm{m}_{\mathrm{v}}<21$ and $\mathrm{z}<0.9$. Data obtained at the Steward Observatory $2.3^{\mathrm{m}}$ and Kitt Peak National Observatory $4^{\mathrm{m}}$ and $2.1^{\mathrm{m}}$ telescopes include $\mathrm{B}, \mathrm{V}, \& \mathrm{R}$ broad band images; narrow band emission line images, low dispersion longslit spectrophotometry covering $3700 \AA$ from $[\mathrm{O} \mathrm{II}] \lambda 3727$ to [O III] $] \lambda 5007$; and high dispersion longslit spectrophotometry around $\mathrm{H} \beta$ \& [O III]. In these proceedings we present the spectroscopic results (Gelderman and Whittle 1994).

The spectra of CSS sources are characterized by strong, high equivalent width emission lines, indicating the presence of a significant amount of ionized gas. The line emission regions for most CSS sources are too compact to be resolved by ground based optical study. Assuming the NLR emission is only associated with the compact radio structure then we derive covering factors which are up to three orders of magnitude higher than values found in typical AGN. However, from our data we cannot confidently distinguish between the youth, frustration, or enhancement scenarios. There is also no conclusive evidence to suggest that the radio jet is directly responsible for the ionization. Emission line flux ratios are typical of gas photoionized by the active nucleus (Veilleux and Osterbrock 1987) and CSS sources fall along the correlation recognized all other powerful radio galaxies and radio 
loud quasars when narrow line emission luminosity is plotted against radio luminosity at $1.4 \mathrm{GHz}$ rest frequency.

Even if the ionization process is not directly related to the jets, kinematic evidence suggests the NLR clouds are accelerated through jet/gas interactions. Our high dispersion spectra of CSS sources reveal [O III] emission line profiles which tend to be very broad and show considerable velocity substructure compared to other classes of radio-loud AGN. In low radio power Seyfert and nearby radio galaxies, these kinematic characteristics have been supported by other evidence for jet/gas interactions (see Whittle 1989 for review). Whittle (1992) compares [O III] linewidths for a large sample of Seyfert galaxies to just those Seyferts which contain relatively luminous $\left(\mathrm{L}_{1.4}>10^{22} \mathrm{~W} \mathrm{~Hz}^{-1}\right)$ linear radio sources. For most Seyferts, the [O III] linewidth is correlated with measures of the gravitational potential of the host galaxy. However, Seyferts with luminous linear radio sources seem to have broader lines, indicating an additional acceleration mechanism caused by outflowing bipolar radio ejecta. The profiles also tend to show more velocity structure than the profiles of other Seyferts, with velocity splitting which can sometimes be directly related to the radio lobes. A comparable situation is observed for objects from our CSS sample as compared to other radio loud galaxies and quasars. We suggest that the CSS radio jets are accelerating the ionized gas, either as frustrated sources or as youthful sources working steadily outward to become extended classical doubles.

Finally, while our data do not allow us to determine why CSS sources are so compact, we do conclude that CSS sources represent a real physical class rather than simply being a collection of objects with various explanations for the powerful yet subgalactic radio structure. The sources in our sample have spectra which share similar qualities and are unlike the spectra of typical active galaxies or quasars. In addition, we find no significant optical distinctions based on either the extent or morphology of the radio structure. Thus we are unable to confirm results from studies of compact symmetric objects.

\section{References}

Fanti, C., Fanti, R., Schilizzi, R.T., Spencer, R.E., Nan Rendong, Parma, P., van Breugel W.J.M., and Venturi, T. 1990, $A A, 231,333$.

Gelderman, R. and Whittle, M. 1994, Ap. J. Supp., $91,491$.

Spencer, R.E., McDowell, J., Charlesworth, M., Fanti, R., Parma, P., and Peacock, J.A. 1989, M.N.R.A.S., 240, 657.

Veilleux, S. and Osterbrock, D.E. 1987, Ap. J. Supp., 63, 295.

Whittle, M. 1992, Ap. J., 387, 109.

Whittle, M. 1989, in ESO Proceedings: Extranuclear Activity in Galaxies, eds. Meurs \& Fosbury. 